\title{
The Development of Medical Record Items: a User-centered, Bottom-up Approach
}

\author{
YoungAh Kim, $\mathrm{PhD}^{1}$, Hangi Park, $\mathrm{MD}, \mathrm{PhD}^{2}$, Hong-Gee Kim, $\mathrm{PhD}^{3}$, Yong Oock Kim, $\mathrm{MD}, \mathrm{PhD}^{4}$ \\ ${ }^{1}$ Department of Medical Informatics, Yonsei University Healthcare System, Seoul; ${ }^{2}$ Department of Cardiovascular Surgery, Yonsei University College of Medi- \\ cine, Seoul; ${ }^{3}$ Biomedical Knowledge Engineering Lab, Seoul National University, Seoul; ${ }^{4}$ Department of Plastic and Reconstructive Surgery, Yonsei University \\ College of Medicine, Seoul, Korea
}

Objectives: Clinical documents (CDs) have evolved from traditional paper documents containing narrative text information into the electronic record sheets composed of itemized records, where each record is expressed as an item with a specific value. We defined medical record (MR) items to be information entities with a specific value. These entities were then used to compile form-based clinical documents as part of an electronic health record system (EHR-s). Methods: We took a reusable bottom-up developmental approach for the MR items, which provided three things: efficient incorporation of the local needs and requirements of the medical professionals from various departments in the hospital, comprehensive inclusion of the essential concepts of the basic elements required in clinical documents, and the provision of a structured means for meaningful data entry and retrieval. This paper delineates our experiences in developing and managing medical records at a large tertiary university hospital in Korea. Results: We collected 63,232 MR items from paper records scanned into 962 CDs. The MR item database was constructed using 13,287 MR items after removing redundant items. During the first year of service users requested changes to be made to 235 (1.8\%) attributes of the MR items and also requested the additional 9,572 new MR items. In the second year, the attributes of $70(0.5 \%)$ of the existing MR items were changed and 3,704 new items were added. The number of registered MR items increased by $72.0 \%$ in the first year and $27.9 \%$ in the second year. Conclusions: The MR item concept provides an easier and more structured means of data entry within an EHR-s. By using these MR items, various kinds of clinical documents can be easily constructed and allows for medical information to be reused and retrieved as data. The success of the use of MR items in a large tertiary university hospital system provides evidence that verifies our approach as being an efficient means of user-oriented and structured data entry, enabling the easy reuse of medical records.

Keywords: Medical Record, Electronic Health Record

Submitted: January 13, 2012

Revised: March 14, 2012

Accepted: March 26, 2012

\section{Corresponding Author}

Yong Oock Kim, MD, PhD

Department of Plastic and Reconstructive Surgery, Yonsei University College of Medicine, 50 Yonsei-ro, Seodaemun-gu, Seoul 120749, Korea. Tel: +82-2-2228-2218, Fax: +82-2-362-5680, E-mail: SGM625@yuhs.ac

This is an Open Access article distributed under the terms of the Creative Commons Attribution Non-Commercial License (http://creativecommons.org/licenses/bync/3.0/) which permits unrestricted non-commercial use, distribution, and reproduction in any medium, provided the original work is properly cited.

(c) 2012 The Korean Society of Medical Informatics

\section{Introduction}

Clinical documents (CDs) serve as a viable means of recording medical data in paper document form with unstructured narrative text information. However, in order to allow for easier and faster writing thereof and to record information in a more systematized manner, CDs have evolved into the form of a record sheet with itemized records collected from various medical fields, each of which can be categorized as an item with a specific value.

CDs are strewn with redundancy and inconsistency in item names, which creates incompatible names for use in inte- 
grated systems such as an electronic health record system (EHR-s). A possible remedy for such obstacles is that CDs should be manufactured with itemized record data with related value(s), which we call medical record (MR) items. The MR item contains not only text or image data, but any type of communicable data. The MR items act as a bridge to link terminology systems and CDs in the implementation of EHR-s. This paper introduces the design concept and structure of the MR items used at a large tertiary university hospital in Korea.

\section{Methods}

The inception and subsequent design and implementation of MR items was part of the design process of the EHR-s at Severance Hospital, a tertiary educational general hospital and the largest hospital among the Yonsei University Healthcare System (http://www.yumc.or.kr) based in Seoul, Korea. The hospital has 64 medical and supportive departments, 2,500 beds, 600 faculty clinicians, and about 8,000 employees.

\section{Concept and Characteristics of MR Item}

The MR item is an itemized record data with related value(s). For example, a 'diagnosis' item in a clinical document can correspond to one or several diseases, and the correspond- ing MR item 'diagnosis' contains entered disease name(s) as a value. The value of MR item can be not only a simple text term, but also images, check boxes, and tables. In this aspect, MR item differs significantly from the text-based terms in the narrative description of a medical record (Figure 1). MR item is either a single element or a collection of MR items called 'a group MR item.' A clinical document is made of various MR items (Figure 2).

\section{Data Collection}

Our first step in data collection was to collect existing paperbased CDs (PbCDs) in use. The collected PbCDs contained not only the PbCDs officially managed by the Medical Record Committee, but also all the available paper records produced during clinical work, such as research protocols of physicians and memos used by the nurses. The initial list of record items was then further processed. First, a hierarchical list of MR items was created. For example, the item 'past history' was often included in admission notes. The item included 'immunization,' 'disease,' and 'operation' as sub-record items. In this case, the item 'immunization' was considered as a second-level item in the admission note; however, it was a first-level item in other clinical documents such as outpatient records in the Pediatric Department. The hierarchical listing of individual MR items allowed for

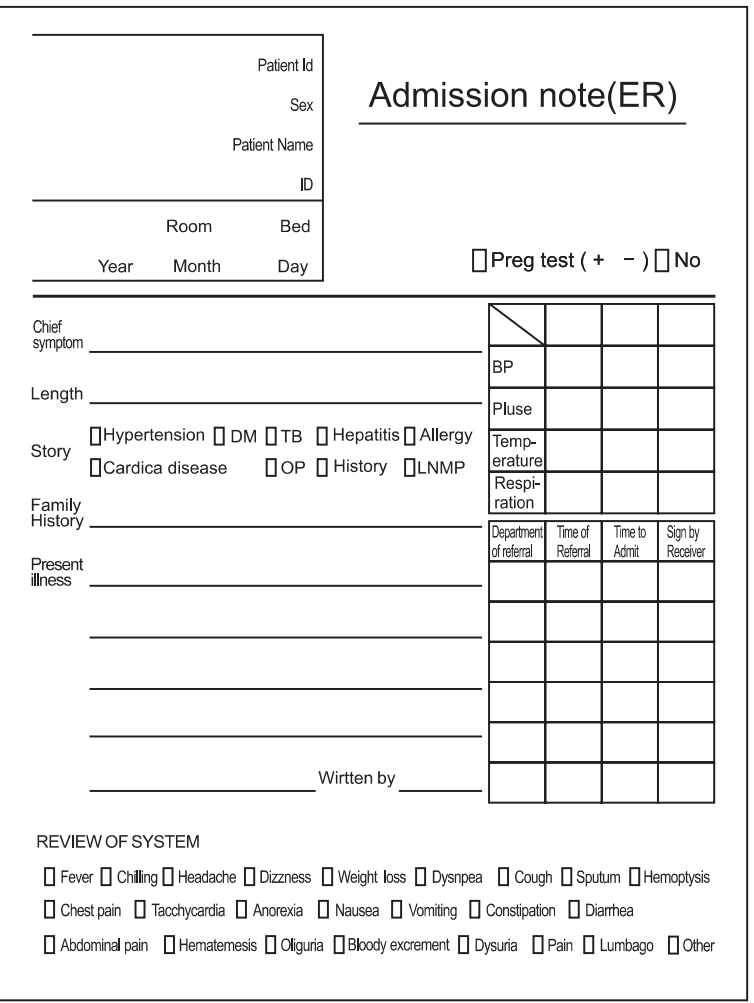

\begin{tabular}{|l|l|l|l|l|l|l|l|l|l|}
\hline Form title & Item & $\begin{array}{c}\text { Level } \\
1\end{array}$ & $\begin{array}{c}\text { Level } \\
2\end{array}$ & $\begin{array}{c}\text { Level } \\
3\end{array}$ & $\begin{array}{l}\text { Level } \\
4\end{array}$ & \multicolumn{1}{|l}{ Type } & Unit & Optional & Remark \\
\hline & $\begin{array}{l}\text { Intern } \\
\text { note }\end{array}$ & $\begin{array}{l}\text { Pregnacy } \\
\text { test }\end{array}$ & & & & $\begin{array}{l}\text { Single } \\
\text { selection }\end{array}$ & Yes, No & \\
\hline $\begin{array}{l}\text { Admission } \\
\text { note (ER) }\end{array}$ & $\begin{array}{l}\text { Chief } \\
\text { symptom }\end{array}$ & Length & & & & Text & & DM, Hypertension,TB \\
\hline & $\begin{array}{l}\text { Past } \\
\text { history }\end{array}$ & & & & & $\begin{array}{l}\text { Multiple } \\
\text { selection }\end{array}$ & DM, Hypertension, TB \\
\hline & Other & & & & Text & & & \\
\hline & Family history & & & & Text & & & \\
\hline & Present illness & & & & Text & & & \\
\hline & Vital sign & BP & & & & $\begin{array}{l}\text { Text } \\
\text { integer }\end{array}$ & mmHg & & \\
\hline & Pluse & & & & $\begin{array}{l}\text { Text } \\
\text { /integer }\end{array}$ & /min & & \\
\hline & Respiration & & & $\begin{array}{l}\text { Text } \\
\text { /integer }\end{array}$ & /min & & \\
\hline & Review of system & & & & $\begin{array}{l}\text { Multiple } \\
\text { selection }\end{array}$ & & Fevere, Chilling, \\
Headache, dizzness
\end{tabular}

Figure 1. Hierarchical list of medical record items. 
more comprehensive utilization of record items. Second, the individual functional characteristics of all MR items were described throughout the hierarchical list. The information taken from other characteristics was likewise added after individually checking the recorded data in PbCDs submitted by clinicians and nurses.

\section{Filtering MR Items}

After the first two collection steps, the collected MR items were filtered in four steps. First, redundant MR items from similar CDs were excluded. And, secondly, same or similar informational MR items from different CDs were excluded. The similarity or identity of MR items was determined on a terminological basis, which took into account functional characteristics identified by clinicians and nurses. An inhouse developed document analyzing tool was used to aid in the filtering process (Figure 3).

The third step was to combine two or more MR items with redundant information. As an example, the MR item 'chief complaint' in inpatient progress notes referred to the same information as the MR item 'chief symptom' found in emergency records; hence, we decided to use the single MR item of 'chief complaint' for both CDs, and accordingly, replaced all instances of 'chief symptom' in the emergency records with 'chief complaint' (Figure 4).

The final filtering process was to identify MR items that carried the same name but referred to different information. Such MR items were split into more than one item, which were more suitable to their actual meanings or functional characteristics. For example, skin color can take on different meanings; it can refer to the skin color of a particular race or a physical finding representing medical conditions such as

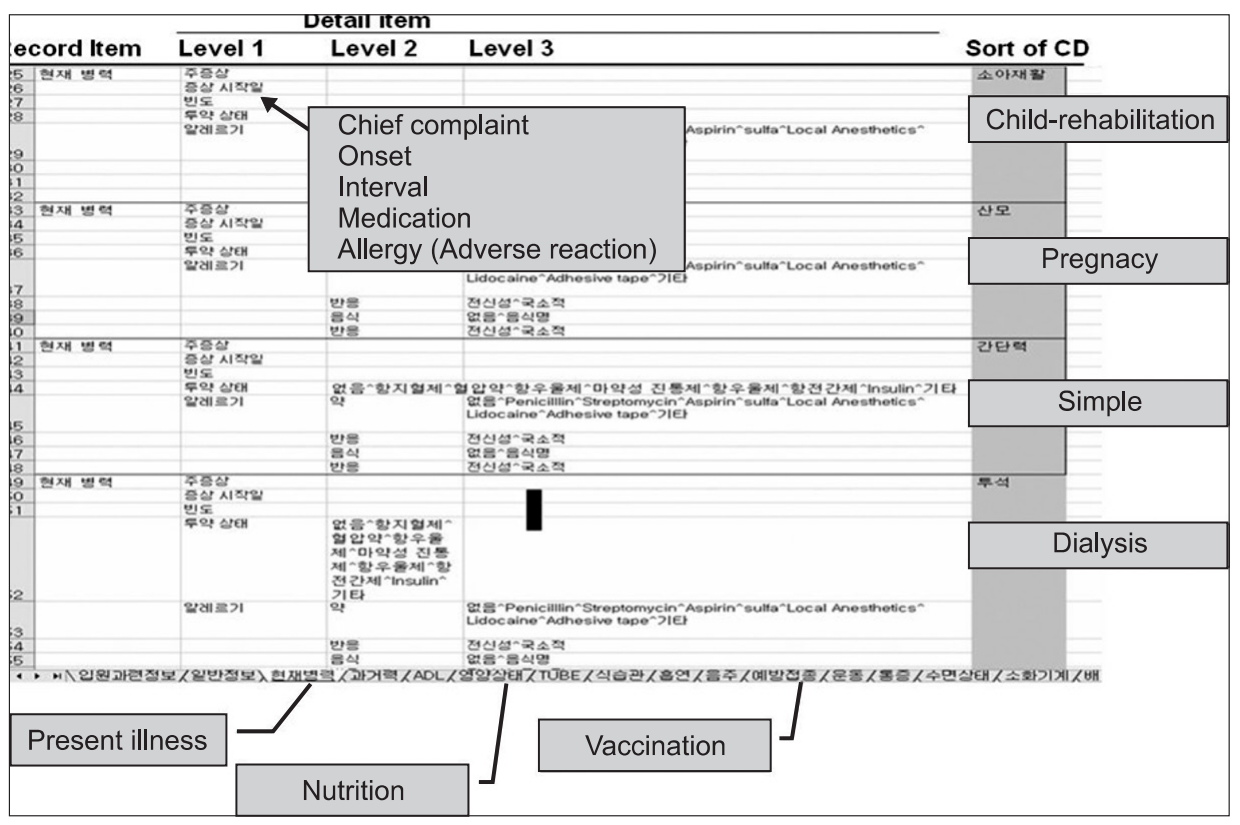

Figure 2. Similarity or identity checking.

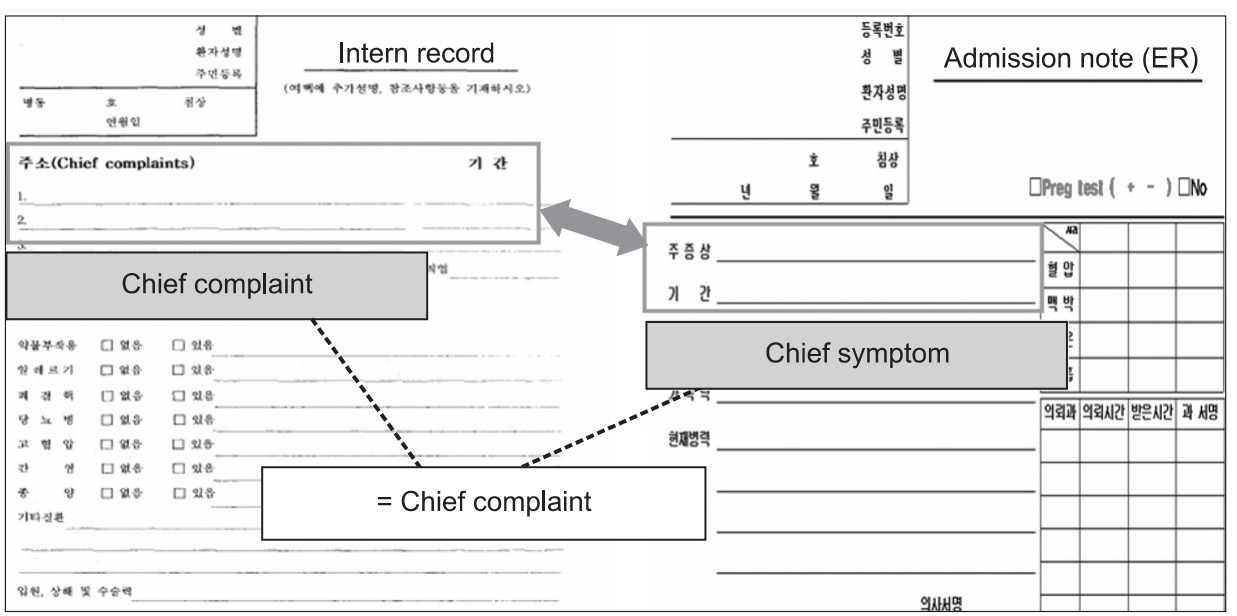

Figure 3. Merge of redundant medical record items into a single item. 
Group MR item (Grid) of 'Physical examination'

- Combination of several MR items constitutively as like MS-excel

- It can also have another classification

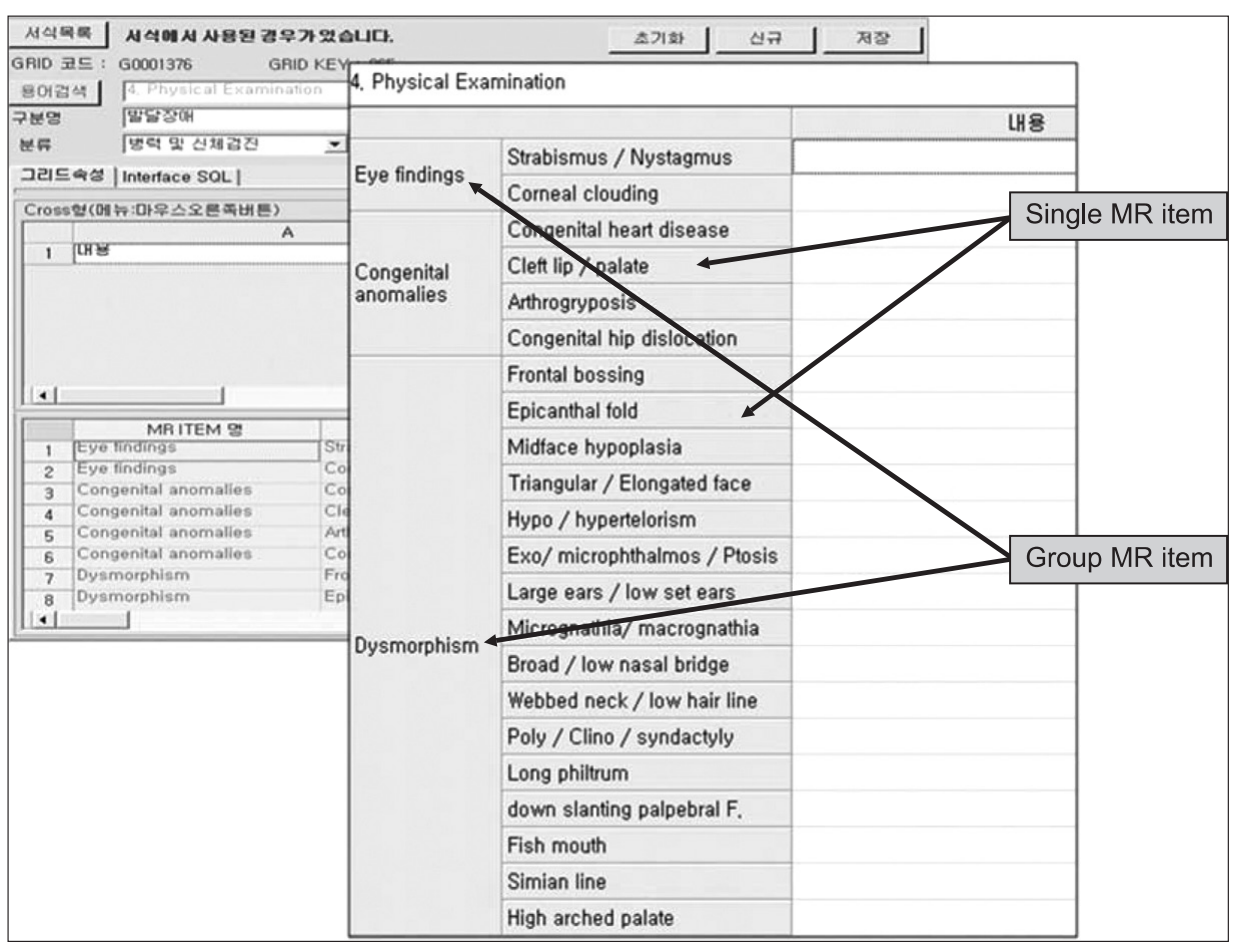

Figure 4. Grid arrangement. MR: medical record.

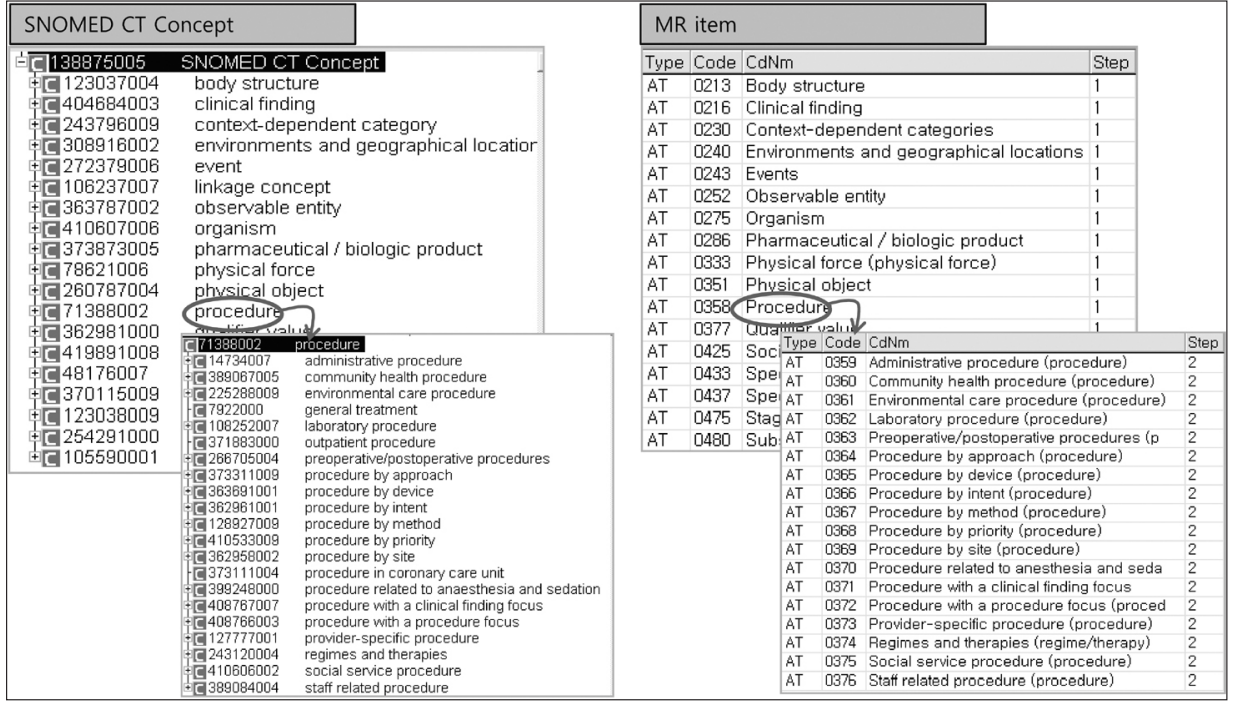

Figure 5. Systematized Nomenclature of Medicine (SNOMED) and terminology attributes. jaundice or pigmentation disorders. In this case, we created two MR items for 'skin color' with different attribute values.

\section{Categorization of Functional Characteristics of MR Items}

After the listing of MR items with their individual characteristics was completed, we examined the characteristics. The categorization thereof was accomplished with the work processes. It became clear that the MR items included characteristics beyond their terminological meanings in CDs. Spe- cifically, the terminology itself could not express whether or not the specific record item was essential to a specific CD. As an example, identification (ID) number and sex are essential record items in almost every $\mathrm{CD}$; however, the meaning of the terms 'ID number' and 'sex' can be interpreted a number of ways. Therefore, we concluded that these were functional characteristics of the MR items, and designated them as 'attributes' (Figure 5). 


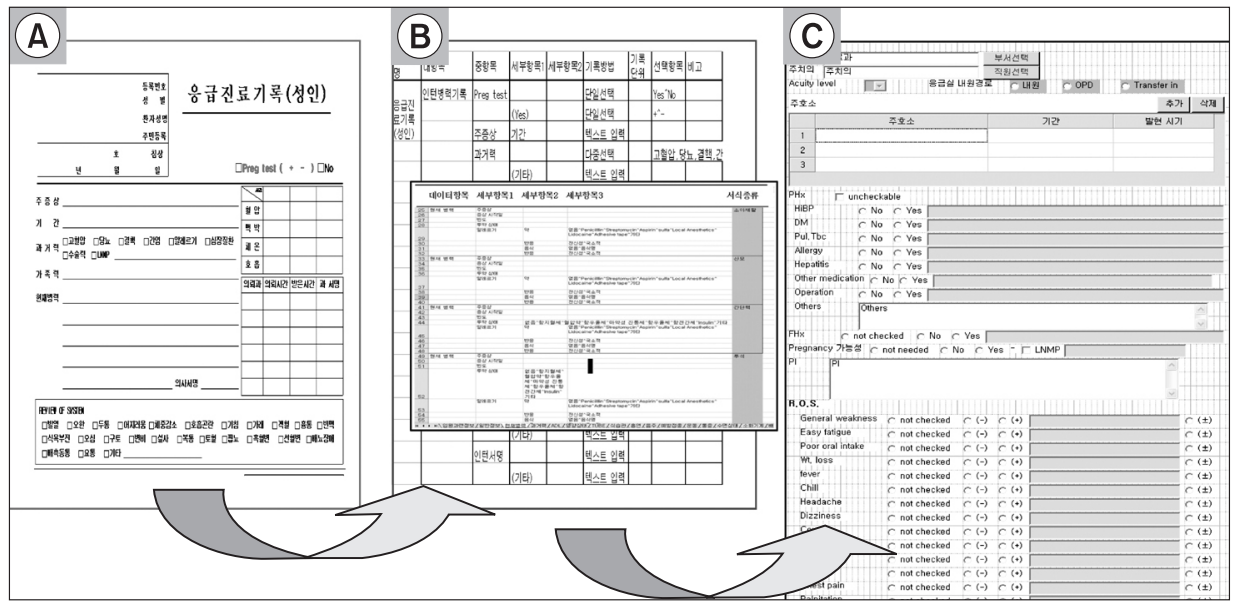

Figure 6. Creation of electronic clinical documents. (A) Paperbased clinical document, (B) collection of medical record items and definition of attribute, (C) clinical document.

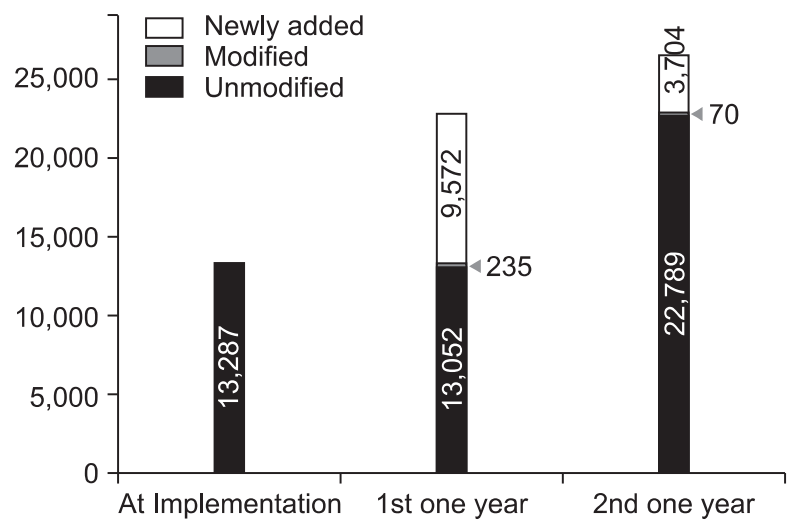

Figure 7. Changes in medical record items over time.

\section{Administration of MR Items}

The end users of the EHR-s were trained with the concept of MR items and proper ways to define MR items (Figure 6). Currently, a designated database administrator manages the MR items. When a new clinical document format is required, the requester(s) and the administrator create the document together by selecting MR items from the database. If the available MR items in the database do not satisfy the requirements of the user, new MR items are defined and subsequently added to the database. When the attributes of MR item are found to be inadequate or require further revision, they are revised.

\section{Results}

\section{Contents of MR Items}

A total of 63,232 record items from $962 \mathrm{PbCDs}$ were gathered at the initial stage of the data collection process. After removing redundancies, 13,287 MR items remained for use and were stored in a database (Figure 7).

There were two types of MR items: single and group items.
A single MR item was the smallest record item which did not have any sub-record item. A group MR item comprised sub-record items. Single MR item was combined to create a group MR item. For example, the 'physical examination' item on an admission note can have multiple record items, such as 'head,' 'neck,' 'trunk,' etc. And the 'head,' 'neck,' and 'trunk' MR items are all single items, which are combined to create the group MR item 'physical examination.' A group MR item is a module (a group of single MR items) in a CD. A group MR item cannot have attribute values of its own, but instead maintains the values of the attributes that belong to the constituent MR items. There are two types of group MR items: a simple serial arrangement of multiple single MR items, and a grid arrangement that contains both single and group MR items. The grid arrangement can be expressed as a tree structure.

\section{Functional Characteristics-Attributes of MR Items}

A single MR item has its own attribute value(s). There are five distinct types of attributes: the terminological attribute, data type attribute, management attribute, sharing attribute, and validity attribute.

The terminological attribute defines the terminological meaning and its terminological relation to international terminology systems such as Systematized Nomenclature of Medicine-Clinical Terms (SNOMED-CT), International Statistical Classification of Diseases and Related Health Problems 10th revision (ICD-10), clinical modification of ICD 9th revision (ICD-9CM), and nursing terminologies. We adopted SNOMED-CT to classify MR items. The terminological attribute has three levels of hierarchical classification as its sub-attribute groups. Levels 1 and 2 are the same as the level 1 concept ID of SNOMED-CT and the level 2 concept ID of SNOMED-CT, respectively. The level 3 subdivision is 
referenced by its own terminological meaning.

The data type attribute defines the input data type of MR item. And the supported data types include a combo box, check button, radio button, image, genealogical chart, yearmonth-day (yyyy-mm-dd), year-month-day-hour-min (yyyy-mm-dd hr:min), hour-min (hr:min), hour-minsecond (hr:min:sec), and text.

The management attribute defines the restrictive or essential aspects of MR item, such as whether an item is an essential object of a record. We assigned seven sub-attributes under the management attribute to assure the quality of medical information: essentiality attribute, completeness attribute, substantiality attribute, reusability attribute, database storage attribute, sharing attribute, and validity attribute.

A new sub-attribute can be added when necessary. The essentiality attribute denotes whether a record item should have a value at the time of its storage. The substantiality attribute is used to evaluate the correctness of the data entered, although it should be noted that the quality of the entered data cannot be checked, even though the item is an essential item to any given CD. The completeness attribute denotes whether the record item is responsible for checking the deadline date of a CD. The reusability attribute denotes whether the item's value can automatically be reused in a new CD for the same patient. The database storage attribute describes whether the value of an item should be stored in the database.

The sharing attribute defines the sharing factor of the entered value of a MR item, and can be thought of as a kind of linked phrase directed to the location of existing data. This attribute has no sub items. The validity attribute defines the history of the creation, alteration, and deletion time of MR item, which allows for the tracing of the lifecycle of MR item.

\section{Creation of Electronic CDs (ECDs) and Implementation of the EHR-s}

Initially, 1,200 ECDs were created by combining MR items from a hierarchical pool of items.

\section{Changes to the MR Item Database}

Much has changed concerning MR items during the first three years in service. Initially, 13,287 MR items were registered to the database, and during the first year of service users requested changes to be made to 235 (1.8\%) attributes of MR items and also requested the addition of 9,572 new MR items. In the second year, the attributes of $70(0.5 \%)$ existing MR items were changed and 3,704 new items were added. The number of registered MR items increased by $72.0 \%$ in the first year and $27.9 \%$ in the second year.

\section{Discussion}

The long term effectiveness of an EHR-s depends on how much comparable and granular data can be produced through controlled and structured entry of health terminologies, and to what extent medical personnel are willing to keep up with data input and reuse data from the system [1]. Finding an efficient way to connect the terminology system with structured data input of clinical documents posed great difficulties. We first tried to discover and infer the clinician's requirements from medical documentation through the examination of paper-based documents. During the examination of the documents, we tried to find medical records of high quality. Good medical documentation relies on accuracy and time [2]. However, due to insufficient time allowed to clinicians to care for their patients, accurate documentation has become a difficult goal to achieve. Indeed, the lack of time for recording data has forced clinicians to complete medical data recording as fast as possible. Clinicians tend to believe that writing too much is just as bad as writing too little [3], and as a result, they have developed many kinds of simplifications for medical data recording. Our data showed that over $65 \%$ of the information in the PbCDs we analyzed were IRTVs, not narrative data, which were strewn with shorthand descriptions.

The filtering process was crucial in that it ensured consensus and motivation for standardized terms among clinicians who had enjoyed the freedom of using terms as best fit their personal needs. The MR item and its value are stored both in an XML file [4] and in the database. The XML file contains the MR item, whereas the item value is stored into the database. The storage of MR items in XML files allows the integrity of medical records to be maintained. On the other hand, the database enables item values to be reused.

MR items can be considered an interface terminology; however, they exhibit somewhat different characteristics from the terminology system. The main difference is its use in CDs and the seven functional attributes. It is evident that a controlled healthcare terminology, such as SNOMED$\mathrm{CT}$, is an important to medical data compatibility in EHR-s. Some reports [5-8] showed that SNOMED-CT covers more than $80 \%$ of terms concerning problems and diagnosis via compositional post-coordination. We used SNOMED-CT and other international terminology systems when mapping our terminologies. When a new MR item is added, the mapping thereof is accomplished by searching pre-coordinated terms and post-coordination terms by our reviewers [9-11]. In our institute, $30 \%$ of all terms are recorded in Korean and the need for an easier, faster data entry tool was strong 
among clinicians, due to time-intensive work in outpatient departments. Also, clinicians tended to use only a small collection of terms in their daily practice, and these terms included local terms used.

The designation of input data type on each record item is important for data storage and reuse, induction of consistent data input, and support for various user interfaces. The recent Health Level 7 clinical document architecture (HL7 CDA) includes a new set of data types, which necessitates the involvement of data types of records, such as text, images, sound, and other multimedia content into ECDs, as a defined and complete information object [12].

The use of the presently described EHR-s based on the application of MR items has been successful, due to its high adaptability to the needs of the clinicians and nurses in our institution. During the first year of service, the number of MR items increased by $72 \%$. This reflected an increased need from end users for more accurate and wider expression power of records during the acute adaptation period. The relatively high number of modifications in the first year represented an urgent need for the hospital to adjust itself to the way medical information was produced and used. Less was changed in the following years, indicating increased stability of the system.

Effective maintenance of the system is of paramount importance. When MR item has not been used in ECDs for a considerable period of time, it is deemed obsolete; and when two items are used as the same MR item but show a great difference in usage, the less popular item is considered obsolete. Clinicians have been continually educated on the concept of MR items, registration processes of MR items, and production rules for CDs. We believe that our educational programs have greatly contributed to the rapid standardization and normalization of the system by fostering consensus and adoption from the end users.

To date, the MR items have been implemented as part of the Korean National Police Hospital (NPH) EHR-s. Initially, a total of 24,973 MR items were exported in the NPH EHR-s at the end of October, 2006. By the end of 2006, the NPH had modified 37 items, and created 379 new items. During 2007, 340 items were modified, and 1,862 new items were created. And in 2008, 475 items were modified, and 433 new items were created. In short, most of the MR items from Severance Hospital were used as they were offered, which we assumed to be proof of the interoperable nature of the MR items.

MR items are different from other medical records in that they are not a terminology system or a CDA. They instead combine the essential features of both terminology systems and CDAs in a practical, bottom-up approach. We believe that MR items have played an important role in ensuring a high-quality EHR-s, by providing an improved medical recording mechanism, data normalization, and effective information sharing among EHR-s components

In conclusion, MR items provide an easier and more structured means of data entry as an EHR-s. They facilitate efficient construction of various kinds of clinical documents while enabling the easy to reuse of medical information. The success of MR items at a large tertiary university hospital has validated the usability of our bottom-up approach as a means of user-oriented and structured data entry, enabling the easy to reuse of the medical record.

\section{Conflict of Interest}

No potential conflict of interest relevant to this article was reported.

\section{Acknowledgments}

This work was supported by the grant No. 10033247 from the Ministry of Knowledge Economy, Korea.

\section{References}

1. Eduljee A, Rocha RA. Practical evaluation of clinical guideline document models. AMIA Annu Symp Proc 2003;2003:836.

2. Soto CM, Kleinman KP, Simon SR. Quality and correlates of medical record documentation in the ambulatory care setting. BMC Health Serv Res 2002;2:22.

3. Tully MP, Cantrill JA. Insights into creation and use of prescribing documentation in the hospital medical record. J Eval Clin Pract 2005;11:430-7.

4. The World Wide Web Consortium (W3C). Extensible markup language (XML) 1.0. Cambridge (MA): W3C; 1998. Technical report no.: REC-xml-19980210.

5. Elkin PL, Brown SH, Husser CS, Bauer BA, WahnerRoedler D, Rosenbloom ST, Speroff T. Evaluation of the content coverage of SNOMED CT: ability of SNOMED clinical terms to represent clinical problem lists. Mayo Clin Proc 2006;81:741-8.

6. Wasserman H, Wang J. An applied evaluation of SNOMED CT as a clinical vocabulary for the computerized diagnosis and problem list. AMIA Annu Symp Proc 2003; 2003:699-703. 
7. Elevitch FR. SNOMED CT: electronic health record enhances anesthesia patient safety. AANA J 2005;73:361-6.

8. Nash SK. Nonsynonymous synonyms: correcting and improving SNOMED CT. AMIA Annu Symp Proc 2003;2003:949.

9. Herbert I, Hawking M. Bringing SNOMED-CT into use within primary care. Inform Prim Care 2005;13:61-4.

10. Elkin PL, Brown SH, Carter J, Bauer BA, WahnerRoedler D, Bergstrom L, Pittelkow M, Rosse C. Guideline and quality indicators for development, purchase and use of controlled health vocabularies. Int J Med Inform 2002;68:175-86.

11. Elkin PL, Brown SH, Lincoln MJ, Hogarth M, Rector A. A formal representation for messages containing compositional expressions. Int J Med Inform 2003;71:89102.

12. Dolin RH, Alschuler L, Beebe C, Biron PV, Boyer SL, Essin D, Kimber E, Lincoln T, Mattison JE. The HL7 clinical document architecture. J Am Med Inform Assoc 2001;8:552-69. 